\title{
Investigation on a giant magnetoelectric effect hexaferrite via neutron scattering techniques
}

\author{
Yan $\mathrm{Wu}^{1}$, Kun Zhai ${ }^{2}$, Wei Tian ${ }^{1}$, Young $\mathrm{Sun}^{2}$, Huibo $\mathrm{Cao}^{1}$ and Fangwei Wang ${ }^{3}$ \\ ${ }^{1}$ Quantum Condensed Matter Division, Oak Ridge National Laboratory, Oak Ridge, TN 37831 \\ ${ }^{2}$ Institute of Physics, Chinese Academy of Science, China \\ ${ }^{3}$ Institute of Theoretical Physics, Chinese Academy of Science, China
}

\begin{abstract}
Y-type hexaferrite $\mathrm{Ba}_{2} \mathrm{Mg}_{2} \mathrm{Fe}_{12} \mathrm{O}_{22}$ was reported recently to have a giant magnetoelectric effect (ME). The magnetic structure of $\mathrm{Ba}_{2} \mathrm{Mg}_{2} \mathrm{Fe}_{12} \mathrm{O}_{22}$ consists of two groups of $\mathrm{L}$ - (large moment) and $\mathrm{S}$ - (small moment) blocks stacking along the $c$-axis direction. The moments align ferrimagnetically in the same block. At zero field, it displays a proper screw magnetic structure with an incommensurate wavevector $\boldsymbol{k}$ along the $c$-axis below a ferrimagnetic-antiferromagnetic (FM-AFM) transition (195 K) and then transforms to a longitudinal conical phase below $50 \mathrm{~K}$. Applying a small magnetic field, the material displays polarization in the conical phase. When doped with $\mathrm{Sr}$, the material keeps its sensitivity to field while the ME coefficient is greatly enhanced with Sr doping. The transition temperatures are largely elevated and FM-AFM transition temperature goes above room. Meanwhile, temperature dependent neutron diffraction investigation shows new commensurate AFM peaks emerging at heavy Sr doped sample. However, size effect is not sufficient to explain such changes upon $\mathrm{Sr}$ doping. Comparison study of the crystal and magnetic structure of the doped samples is performed to identify the exquisite atomic position changes and the moment interaction picture differences with doping.
\end{abstract}

